Supporting Information for

\title{
Using Hybrid Organic-Inorganic Surface Technology to Mitigate Analyte Interactions with Metal Surfaces in UHPLC
}

Mathew DeLano, Thomas H. Walter*, Matthew A. Lauber, Martin Gilar, Moon Chul Jung †', Jennifer M. Nguyen, Cheryl Boissel, Amit V. Patel, Andrew Bates-

\author{
Harrison and Kevin D. Wyndham
}

Waters Corporation, 34 Maple Street, Milford, MA

† Current address: Enanta Pharmaceuticals Inc, 500 Arsenal Street, Watertown, MA

*Corresponding Author

Tel.: (508) 498-9633. Email: tom_walter@waters.com.

\section{Table of Contents}

Page S2 - Figure S1: ATP Recovery for Standard and Hybrid Surface Capillary Tubing Page S3 - Table S1: Gradient conditions for LC/MS/MS of ATP, ADP, AMP and adenosine

Page S4 - Table S2: SRM conditions for LC/MS/MS of ATP, ADP, AMP, and adenosine

Page S5 - Figure S2. Calibration curves for ATP and ADP obtained using LC/MS/MS

Page S6 - Figure S3. Calibration curves for AMP and adenosine obtained using LC/MS/MS

Page S7 - Table S3. Calibration ranges and correlation coefficients (r) for ATP, ADP, AMP and adenosine obtained using LC/MS/MS

Page S8 - Figure S4: Mass spectra of enolase T37 Using Standard and Hybrid Surface Columns 
Figure S1: ATP Recovery for Standard and Hybrid Surface $100 \mu \mathrm{m}$ x 368 mm MP35N Capillary Tubing

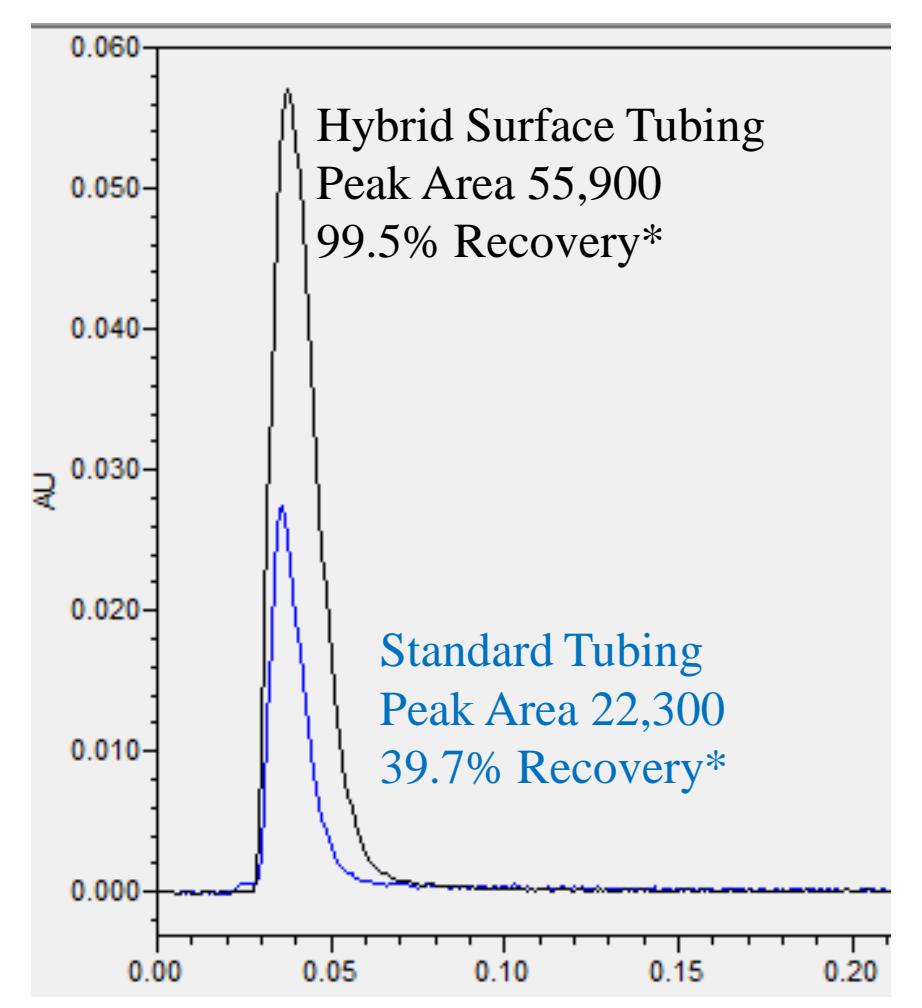

* Relative to a $100 \mu \mathrm{m}$ x $406 \mathrm{~mm}$ PEEK tube
ACQUITY I-Class with PEEK needle and $10 \mu \mathrm{L}$ sample loop with HST

\section{ACQUITY TUV Detector}

No column

Mobile Phase: $10 \mathrm{mM}$ ammonium acetate $\mathrm{pH} 6.5$ Sample: $0.05 \mathrm{mg} / \mathrm{mL}$ ATP in water Injection Volume: $0.2 \mu \mathrm{L}$

Flow Rate: $0.2 \mathrm{~mL} / \mathrm{min}$

Detection Wavelength : $260 \mathrm{~nm}$

Sample Temperature: $4^{\circ} \mathrm{C}$

Column Manager Temperature: $30^{\circ} \mathrm{C}$ 
Table S1: Gradient conditions for LC/MS/MS of ATP, ADP, AMP and adenosine.

- Mobile Phase A: aqueous 10 mM ammonium acetate, pH 6.8 (0.2\% acetonitrile)

- Mobile Phase B: acetonitrile

- Gradient

\begin{tabular}{ccccc}
\hline Time & Flow rate & \%A & \% B & Curve \\
\hline Initial & $0.5 \mathrm{~mL} / \mathrm{min}$ & 99.5 & 0.5 & - \\
0.1 & $0.5 \mathrm{~mL} / \mathrm{min}$ & 99.5 & 0.5 & 6 \\
0.2 & $0.5 \mathrm{~mL} / \mathrm{min}$ & 92.0 & 8.0 & 6 \\
0.7 & $0.5 \mathrm{~mL} / \mathrm{min}$ & 92.0 & 8.0 & 6 \\
0.8 & $0.5 \mathrm{~mL} / \mathrm{min}$ & 70.0 & 30.0 & 6 \\
0.9 & $0.5 \mathrm{~mL} / \mathrm{min}$ & 70.0 & 30.0 & 6 \\
1.0 & $0.5 \mathrm{~mL} / \mathrm{min}$ & 99.5 & 0.5 & 6 \\
2.0 & $0.5 \mathrm{~mL} / \mathrm{min}$ & 99.5 & 0.5 & 11 \\
\hline
\end{tabular}


Table S2: SRM conditions for LC/MS/MS of ATP, ADP, AMP, and adenosine.

\begin{tabular}{ccccc}
\hline & MW & Transition & $\begin{array}{c}\text { Cone } \\
\text { Voltage } \\
(\mathbf{V})\end{array}$ & $\begin{array}{c}\text { Collision } \\
\text { Energy } \\
(\mathbf{e V})\end{array}$ \\
\hline ATP & 507.18 & $505.96>158.84$ & 30 & 30 \\
ADP & 427.20 & $425.98>134.00$ & 48 & 22 \\
AMP & 347.22 & $346.00>134.00$ & 50 & 30 \\
\hline Adenosine & 267.24 & $266.02>134.00$ & 32 & 18 \\
\hline
\end{tabular}



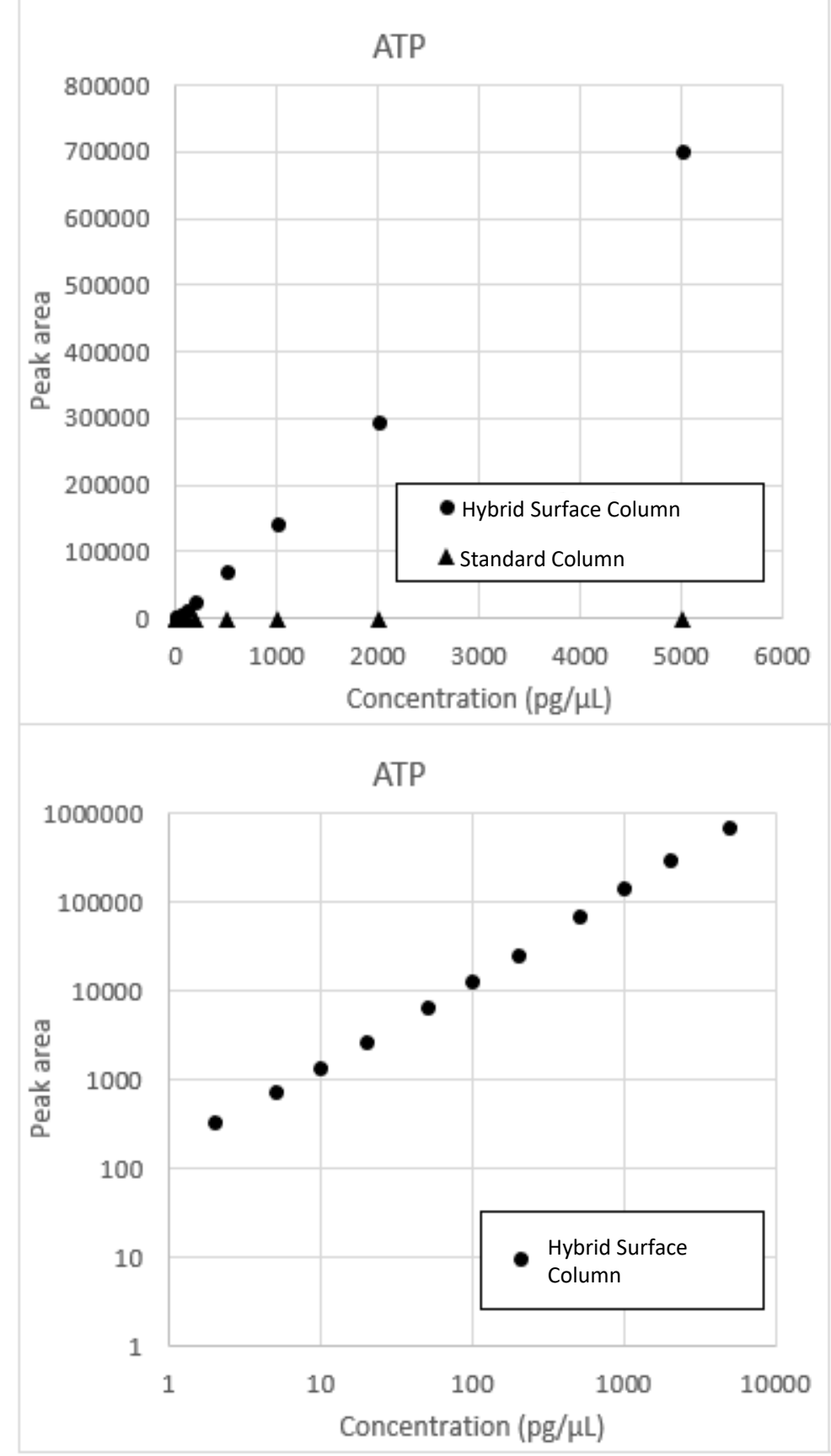
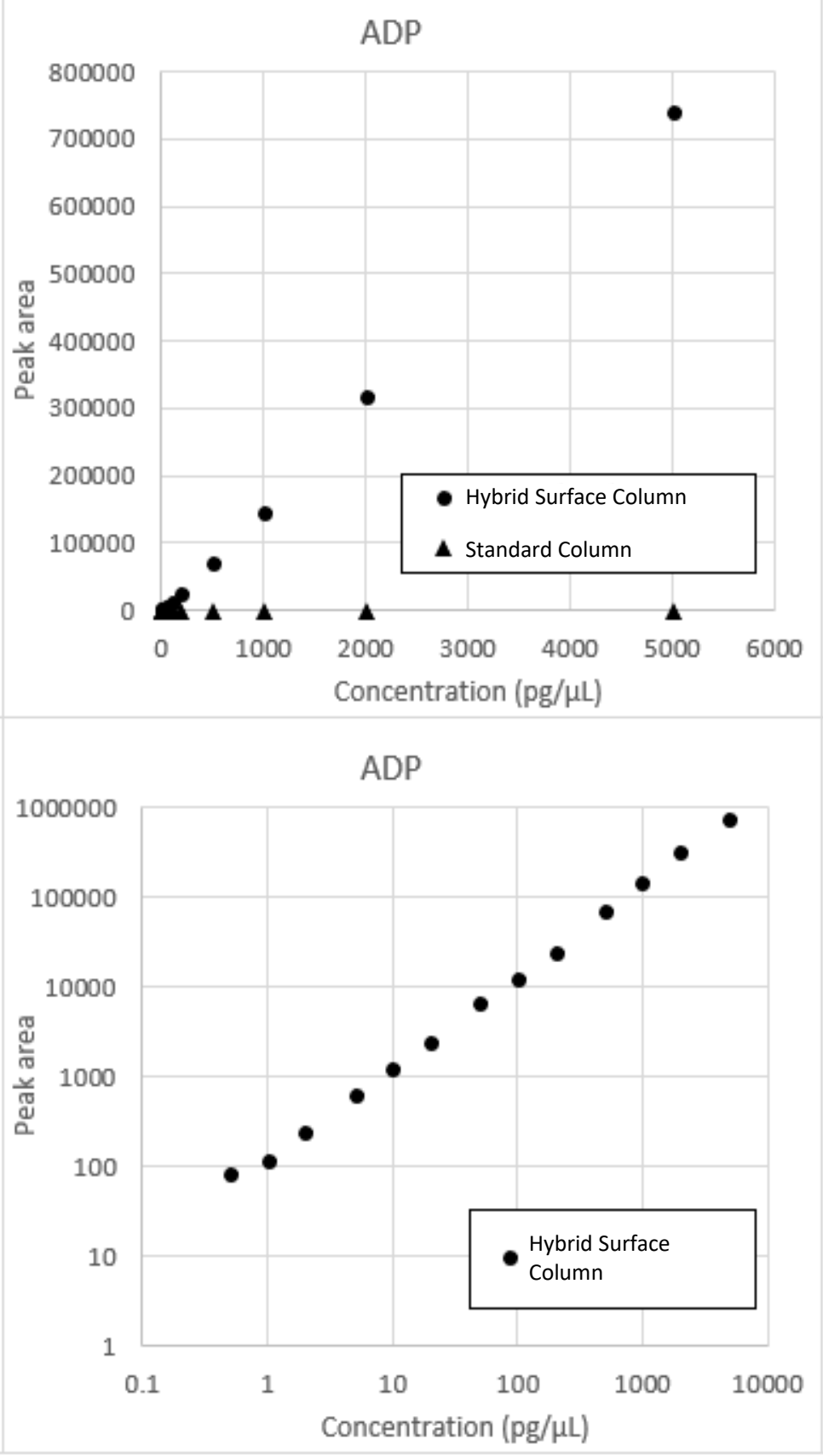

Figure S2. Calibration curves for ATP and ADP obtained using LC/MS/MS with a standard ACQUITY UPLC HSS T3 $1.8 \mu \mathrm{m}$ $2.1 \times 50 \mathrm{~mm}$ column or a column constructed using hybrid surface technology, containing the same stationary phase. Top row: linear $\mathrm{x}$ axes, bottom row: logarithmic $\mathrm{x}$ axes. The injection volume was $1 \mu \mathrm{L}$. 

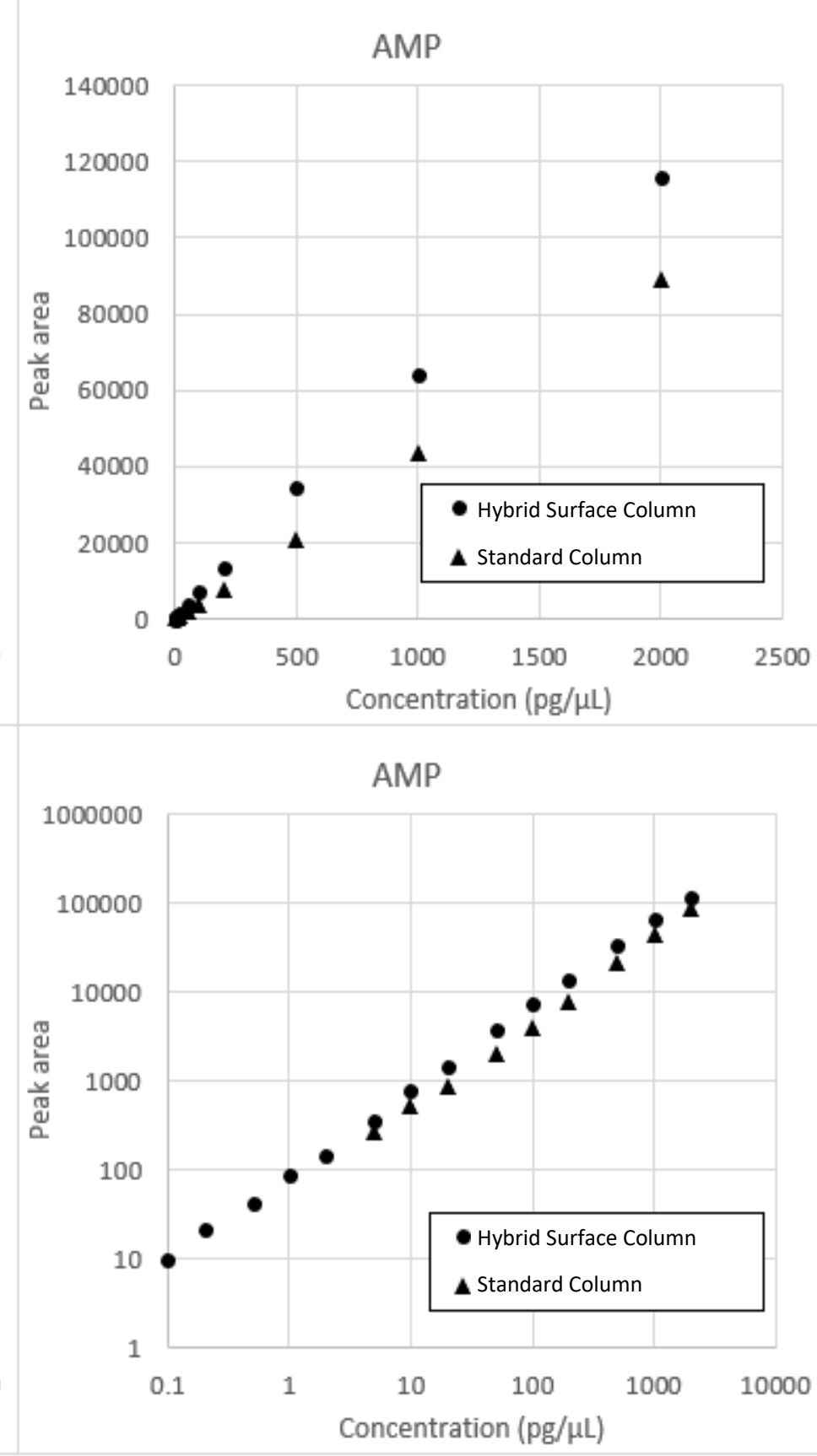
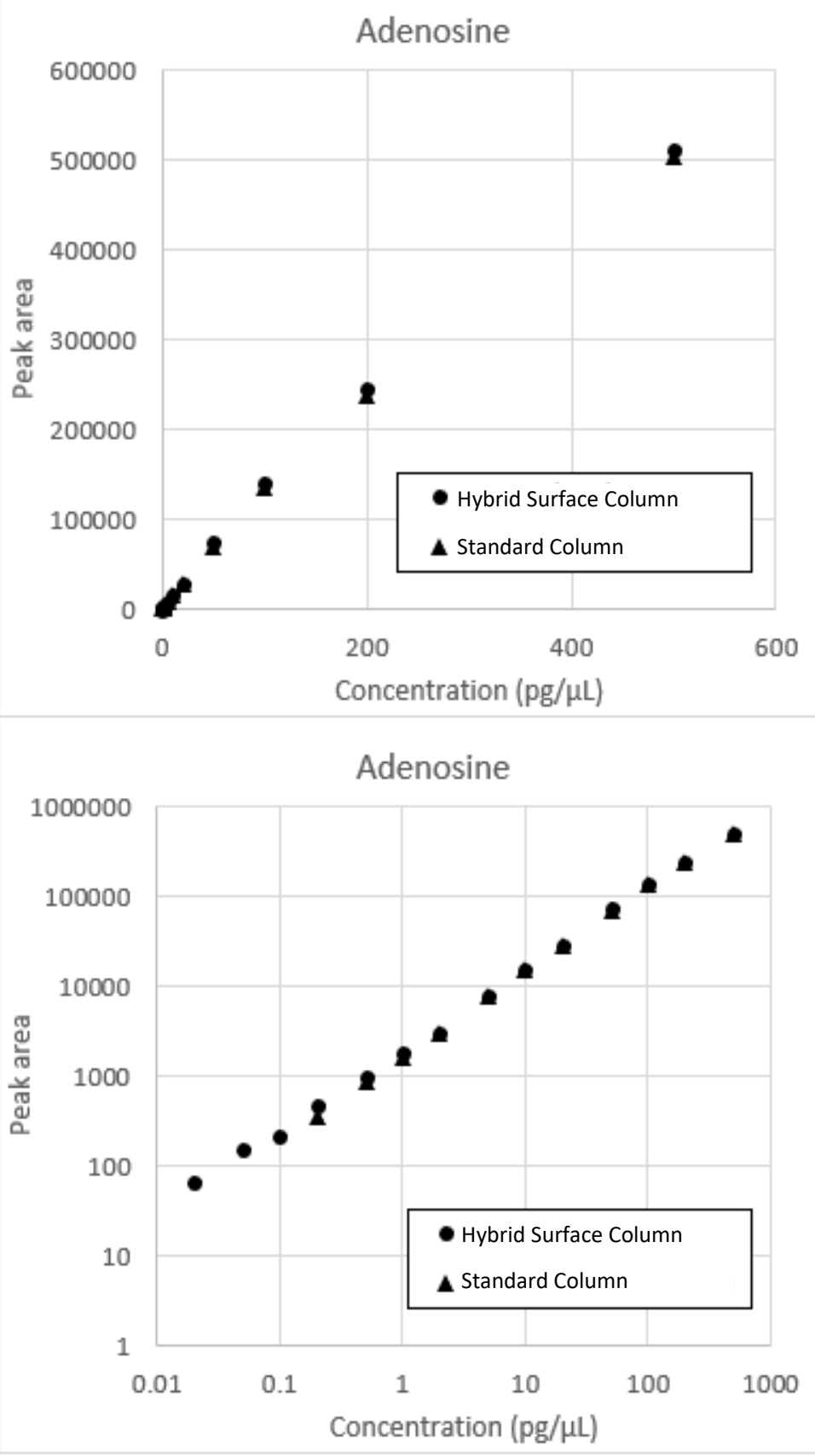

Figure S3. Calibration curves for AMP and adenosine obtained using LC/MS/MS with a standard ACQUITY UPLC HSS T3 $1.8 \mu \mathrm{m}$ $2.1 \times 50 \mathrm{~mm}$ column or a column constructed using hybrid surface technology, containing the same stationary phase. Top row: linear $\mathrm{x}$ axes, bottom row: logarithmic $\mathrm{x}-$ axes. The injection volume was $1 \mu \mathrm{L}$. 
Table S3. Calibration ranges and correlation coefficients ( $r$ ) for ATP, ADP, AMP and adenosine obtained using LC/MS/MS with a standard ACQUITY UPLC HSS T3 $1.8 \mu \mathrm{m} 2.1 \times 50 \mathrm{~mm}$ column or a column constructed using hybrid surface technology, containing the same stationary phase. The injection volume was $1 \mu \mathrm{L}$.

\begin{tabular}{|c|c|c|c|c|c|c|c|c|}
\hline & \begin{tabular}{|c} 
ATl \\
Calibration \\
Range
\end{tabular} & $r$ & $\begin{array}{c}\text { AD } \\
\text { Calibration } \\
\text { Range }\end{array}$ & $r$ & $\begin{array}{c}\text { AM } \\
\text { Calibration } \\
\text { Range }\end{array}$ & $r$ & $\begin{array}{l}\text { Adenos } \\
\text { Calibration } \\
\text { Range }\end{array}$ & $\begin{array}{r}\text { ne } \\
r\end{array}$ \\
\hline $\begin{array}{l}\text { Hybrid Surface } \\
\text { Column }\end{array}$ & $\begin{array}{c}2 \mathrm{pg} / \mu \mathrm{L}- \\
5 \mathrm{ng} / \mu \mathrm{L}\end{array}$ & 0.9996 & $\begin{array}{c}500 \mathrm{fg} / \mu \mathrm{L}- \\
5 \mathrm{ng} / \mu \mathrm{L}\end{array}$ & 0.9986 & $\begin{array}{c}100 \mathrm{fg} / \mu \mathrm{L}- \\
2 \mathrm{ng} / \mu \mathrm{L}\end{array}$ & 0.9972 & $\begin{array}{l}20 \mathrm{fg} / \mu \mathrm{L}- \\
0.5 \mathrm{ng} / \mu \mathrm{L}\end{array}$ & 0.9895 \\
\hline Standard Column & not detected & 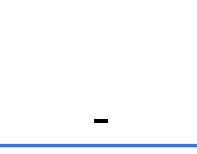 & not detected & 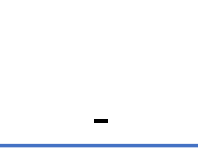 & $\begin{array}{c}5 \mathrm{pg} / \mu \mathrm{L}- \\
2 \mathrm{ng} / \mu \mathrm{L}\end{array}$ & 0.9977 & $\begin{array}{c}200 \mathrm{fg} / \mu \mathrm{L}- \\
0.5 \mathrm{ng} / \mu \mathrm{L}\end{array}$ & 0.9908 \\
\hline
\end{tabular}


A

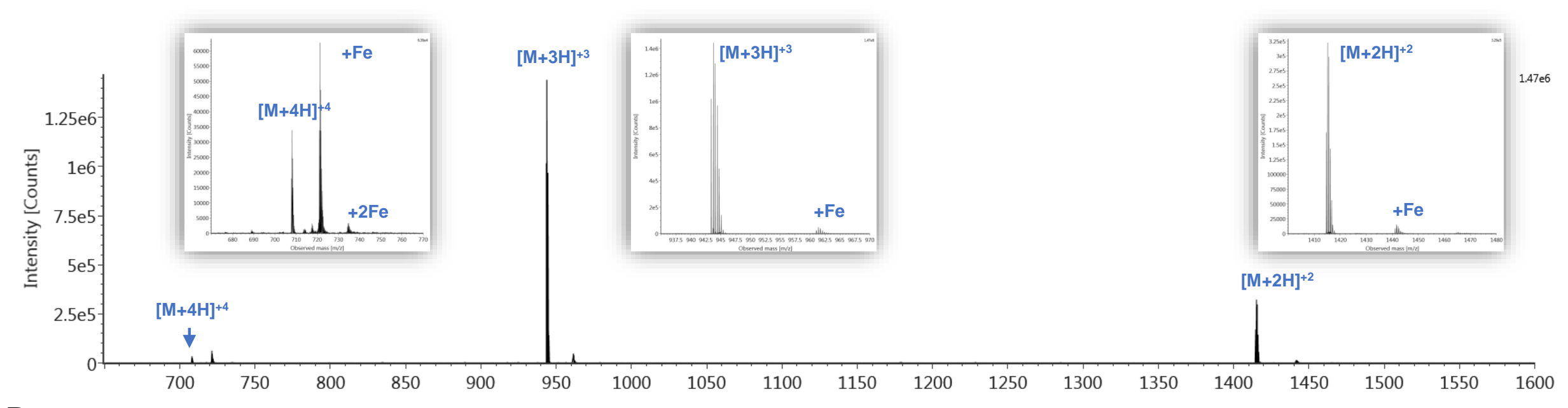

B

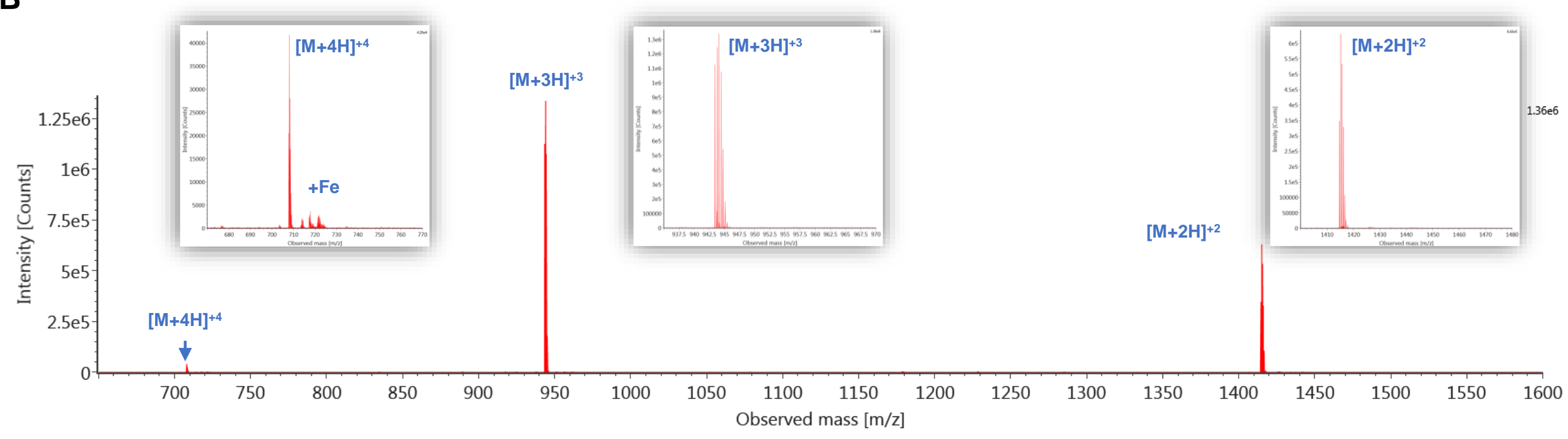

Figure S3: Mass spectra of enolase T37 from separations using a standard ACQUITY UPLC CSH C 18 1.7 $\mu \mathrm{m}$ $2.1 \times 50 \mathrm{~mm}$ column (A) or a column constructed using hybrid surface technology, containing the same stationary phase (B). 